\title{
Risk transformations, deductibles, and policy limits
}

Michael R Powers; Larry Y Tzeng

Journal of Risk and Insurance; Sep 2001; 68, 3; ABI/INFORM Global pg. 465

(CThe Journal of Risk and Insurance, 2001, Vol. 68, No. 3, 465-474

\section{Risk Transformations, Deductibles, and Policy Limits}

\author{
Michael R. Powers \\ Larry Y. Tzeng
}

\begin{abstract}
Rothschild and Stiglitz (1970, 1971) pioneered the study of how an increase in risk affects the demand for a risky asset. Gollier (1995) first identified a necessary and sufficient condition for unambiguous comparative statics for demand under transformations of the asset's probability distribution function. In this article, the authors examine the necessary and sufficient conditions for unambiguous comparative statics for insurance demand for coverage deductibles and policy limits, when the loss variable undergoes certain classes of transformations. The authors show that, if a transformation is both mean-preserving above (below) the optimal deductible (policy limit), and probability-preserving above (below) the optimal deductible (policy limit), then the necessary and sufficient condition is given by first-order stochastic dominance below (above) the optimal point.
\end{abstract}

\section{INTRODUCTION}

Rothschild and Stiglitz $(1970,1971)$ pioneered the study of how an increase in risk affects a risk-averse decision maker's demand for a risky asset. Since then, several researchers (Dreze and Modigliani, 1972; Diamond and Stiglitz, 1974; Dionne and Eeckhoudt, 1987; and Briys, Dionne, and Eeckhoudt, 1989) have found conditions on the individual's utility function that can generate unambiguous comparative statics with a mean-preserving transformation (MPT) of the asset's probability distribution function. Others (Eeckhoudt and Hansen, 1980, 1983; Meyer and Ormiston, 1983, 1985; Black and Bulkley, 1989; and Dionne and Gollier, 1992) have found constraints on the increase in risk that can provide clear prediction.

Gollier (1995) first identified a necessary and sufficient condition for unambiguous comparative statics for demand under transformations of an asset's distribution. This least-constraining condition is called "greater central riskiness" in the case of a linear payoff function.

Michael R. Powers is director of the Advanta Center for Financial Services Studies, The Fox School, Temple University, Philadelphia. Larry Y. Tzeng is associate professor of finance, National Taiwan University, Taipei. The authors thank the two anonymous referees and the editor for their helpful comments. A suggestion of the second referee helped shorten the proofs of both Theorem 1 and Theorem 2. 
The context of the present work is most closely related to that of Eeckhoudt, Gollier, and Schlesinger (1991), who studied increases in risk for deductible insurance and found separate sufficient conditions for both increases and decreases in insurance demand. These authors followed upon earlier work by Schlesinger (1981), who provided an analysis of the demand for deductible insurance. More recently, Meyer and Ormiston (1999) have provided further analysis of deductible insurance, with particular attention to second-order condition issues.

In the present research, models of both insurance deductibles and policy limits are studied. Like Gollier (1995), the authors develop necessary and sufficient conditions for unambiguous comparative statics in both cases.

For insurance deductibles, it is found that if the transformation of the loss distribution preserves the mean, as well as the total probability, above the optimal deductible, then the necessary and sufficient condition is given by first-order stochastic dominance below this point. Alternatively, for policy limits, the authors find that if the transformation of the loss distribution preserves the mean, as well as the total probability, below the optimal policy limit, then the necessary and sufficient condition is first-order stochastic dominance above this point.

\section{The Case of Deductibles}

Insurance deductibles are used frequently in primary property, liability, and health insurance, as well as excess-of-loss and stop-loss reinsurance. The purpose of the deductible mechanism is generally to reduce problems with moral hazard by requiring that the insured take some responsibility for loss payments. Deductibles are also used to reduce the insurer's expense loading by eliminating the claim settlement expenses associated with smaller claims, for which settlement expenses are disproportionately large.

Assume that a risk-averse insured with initial wealth $W$ and (twice-differentiable, increasing, and concave downward) utility function $u(\bullet)$ faces a random loss $X \in[0, L]$, where $X \sim F(\bullet)$. Let $p$ denote the price of insurance coverage and $\delta \leq L$ denote the straight deductible purchased on a direct indemnity policy; i.e., the insured pays a premium $p$ for an indemnity benefit $\operatorname{Max}\{X-\delta, 0\}$. It then follows that the insured's final wealth is given by $z(X)=W-X+\operatorname{Max}\{X-\delta, 0\}-p$.

Now assume that $p$ is actuarially based with profit and expense loading $\theta$ (i.e., $\left.p=(1+\theta) E\left[\operatorname{Max}\left\{X-\delta^{*}, 0\right\}\right]\right)$ and that the risk-averse insured chooses an optimal insurance deductible $\delta^{*}$ to maximize his or her expected utility $E[u(z(X))]$. One then seeks $\delta *$ to maximize

$$
H(\delta ; u, F, z)=\int_{0}^{L} u(z(x)) d F(x)=\int_{0}^{\delta} u(W-x-p) d F(x)+\int_{\delta}^{L} u(W-\delta-p) d F(x)
$$

such that $p=(1+\theta) E[\operatorname{Max}\{X-\delta, 0\}]=(1+\theta)\left\{\int_{\delta}^{L} x d F(x)-\delta[1-F(\delta)]\right\}$, and the optimal deductible can be determined by the first-order condition 


$$
\begin{aligned}
H^{\prime}\left(\delta^{*} ; u, F, z\right) & =\int_{0}^{\delta^{*}}\left(-\frac{d p}{d \delta^{*}}\right) u^{\prime}(W-x-p) d F(x) \\
& +\int_{\delta^{*}}^{L}\left(-1-\frac{d p}{d \delta^{*}}\right) u^{\prime}\left(W-\delta^{*}-p\right) d F(x)=0,
\end{aligned}
$$

where $\frac{d p}{d \delta^{*}}=-(1+\theta) \operatorname{Pr}\left\{X>\delta^{*}\right\}$.

In developing the results, attention is restricted to transformations of the loss distribution function that preserve the mean, as well as the total probability, above the optimal deductible. The primary reason for considering MPTs above the deductible is that this is the most natural ceteris paribus condition for the study of how changes in the loss distribution affect the demand for insurance. ${ }^{2}$ To see this, consider a transformation that is mean-preserving for the entire loss distribution, but such that the mean for the portion of the distribution above the deductible is substantially increased. In this case, insurance-purchasing behavior may easily be affected more by the increase in the price of the policy than by any change in risk. However, if the transformation is mean-preserving above the deductible, then any change in insurance-purchasing behavior can be attributed directly to a change in expected loss or risk below the deductible.

Theorem 1: If $p=(1+\theta) E\left[\operatorname{Max}\left\{X-\delta^{*}, 0\right\}\right]$ and the transformation $F(\bullet) \rightarrow G(\bullet)$ is both

(i) mean-preserving above $\delta *\left(\right.$ i.e., $\left.\int_{\delta^{*}}^{L} x d G(x)=\int_{\delta^{*}}^{L} x d F(x)\right)$ and

(ii) stochastically equivalent at $\delta *\left[\right.$ i.e., $\left.G\left(\delta^{*}\right)=F\left(\delta^{*}\right)\right]$

then the insured will continue to purchase at least as much insurance if and only if

for all $x \in\left[0, \delta^{*}\right]$.

$$
G(x) \geq F(x)
$$

Proof: See the Appendix.

Note that the definition of an MPT above the deductible, given by Condition (i), is equivalent to the assumption that $E\left[X \mid X>\delta^{*}\right] \operatorname{Pr}\left\{X>\delta^{*}\right\}$ is unchanged by the risk transformation. This definition is different from the assumption that $E\left[X \mid X>\delta^{*}\right]$ remains constant, which might be described as a "conditional MPT." Clearly, Conditions (i) and (ii), taken together, imply that $E\left[X \mid X>\delta^{*}\right]$ is constant; however, the converse is not true.

It can be shown that the second-order condition, $H^{\prime \prime}\left(\delta^{*} ; u, F, z\right)<0$ holds for any $\delta^{*} \in(0, L)$. Details are provided in a technical appendix available from the authors.

${ }^{2}$ Note that Conditions (i) and (ii) of Theorem 1 are similar to the premises of Proposition 1 of Eeckhoudt, Gollier, and Schlesinger (1991) [which makes the additional assumption that $G(x)=F(x)$ for all $\left.x<\delta^{*}\right]$. 
Theorem 1 states that if the risk is transformed in a manner that preserves the mean loss above the deductible and is stochastically equivalent at the deductible, then at least as much insurance will be purchased if and only if the transformation yields a worse risk, in the first-order sense, below the deductible. This theorem is similar to Proposition 3 of Eeckhoudt, Gollier, and Schlesinger (1991), which shows that, for an MPT of the entire loss distribution, and an insured with nonincreasing absolute risk aversion, Condition (3) is sufficient for an insured to continue to purchase at least as much insurance under $G(\bullet)$ as under $F(\bullet)$. The authors' principal departure from this result comes from considering transformations that are mean-preserving above the optimal deductible, rather than for the entire distribution function.

Clearly, transformations of the loss distribution are often exogenous to the deductible selected by the insured, in which case it is not clear that the transformations would necessarily be mean-preserving above the insured's particular deductible. However, one important class of problems in which this condition would hold are those involving statutory or regulatory changes that affect the insured's obligation to pay relatively small losses (i.e., those clearly below the deductible) out of his or her wealth. For example, any change in federal income tax policy that has the effect of restricting the insured's ability to deduct the first $\delta^{\prime}$ dollars of a property-liability loss $X$ (where $\delta^{\prime}<\delta^{*}$ ) from taxable income would satisfy the conditions of Theorem 1.

For cases in which the risk transformation arises from activities that are endogenous to the insured-such as the implementation of loss control, self-insurance, or other risk management programs-it is quite likely that the transformation would depend specifically on the deductible. Models of this latter type generally would require consideration of an additional decision variable reflecting the amount of resources directed to the risk management program; these models are addressed as a focus for future research in the final section

\section{The Case of Policy Limits}

Insurance policy limits are often encountered in primary property, liability, and health insurance, as well as various reinsurance coverages. Unlike deductibles, however, they are generally used to protect the insurer from excessive levels of risk rather than problems of moral hazard or claim settlement expenses.

Let $p$ denote the price of insurance coverage and $\lambda \leq L$ denote the maximum limit purchased on a direct indemnity basis; i.e., the insured pays a premium $p$ for an indemnity benefit $\operatorname{Min}\{X, \lambda\}$. It then follows that the insured's final wealth is given by $z(X)=W-X+\operatorname{Min}\{X, \lambda\}-p$.

Again, it is assumed that $p$ is actuarially based with profit and expense loading $\theta$ (i.e., $\left.p=(1+\theta) E\left[\operatorname{Min}\left\{X, \lambda^{*}\right\}\right]\right)$ and that the risk-averse insured chooses an optimal insurance policy limit $\lambda *$ to maximize his or her expected utility $E[u(z(X))]$. One then seeks $\lambda *$ to maximize

$$
H(\lambda ; u, F, z)=\int_{0}^{L} u(z(x)) d F(x)=\int_{0}^{\lambda} u(W-p) d F(x)+\int_{\lambda}^{L} u(W-x+\lambda-p) d F(x)
$$


such that $p=(1+\theta) E[\operatorname{Min}\{X, \lambda\}]=(1+\theta)\left\{\int_{0}^{\lambda} x d F(x)+\lambda[1-F(\lambda)]\right\}$, and the optimal insurance amount can be determined by the first-order condition

$$
\begin{aligned}
H^{\prime}\left(\lambda^{*} ; u, F, z\right) & =\int_{0}^{\lambda^{*}}\left(-\frac{d p}{d \lambda *}\right) u^{\prime}(W-p) d F(x) \\
& +\int_{\lambda^{*}}^{L}\left(1-\frac{d p}{d \lambda^{*}}\right) u^{\prime}\left(W-x+\lambda^{*}-p\right) d F(x)=0,
\end{aligned}
$$

where $\frac{d p}{d \lambda^{*}}=(1+\theta) \operatorname{Pr}\left\{X>\lambda^{*}\right\} .^{3}$

In developing results, attention is restricted to transformations of the loss distribution function that preserve the mean, as well as the total probability, below the optimal policy limit. In the case of deductibles, it was argued that the assumption of an MPT above the optimal deductible is the most natural ceteris paribus condition for the study of how changes in the loss distribution affect the demand for insurance. In this case, the assumption of an MPT below the optimal policy limit is the corresponding ceteris paribus condition, because, under this assumption, any change in insurancepurchasing behavior can be attributed directly to a change in expected loss or risk above the limit.

Theorem 2: If $p=(1+\theta) E[\operatorname{Min}\{X, \lambda *\}]$, the second-order condition is satisfied, and the transformation $F(\bullet) \rightarrow G(\bullet)$ is both

(i) mean-preserving below $\lambda *\left(\right.$ i.e., $\left.\int_{0}^{\lambda^{*}} x d G(x)=\int_{0}^{\lambda^{*}} x d F(x)\right)$ and

(ii) stochastically equivalent at $\lambda *\left(\right.$ i.e., $G\left(\lambda^{*}\right)=F(\lambda *)$ )

then the insured will continue to purchase at least as much insurance if and only if

$$
G(x) \leq F(x)
$$

for all $x \in\left[\lambda^{*}, L\right]$.

Proof: See the Appendix.

Note that the definition of an MPT below the policy limit, given by Condition (i), is equivalent to the assumption that $E\left[X \mid X<\lambda^{*}\right] \operatorname{Pr}\left\{X<\lambda^{*}\right\}$ is unchanged by the risk transformation. This definition differs from the "conditional MPT" assumption that $E\left[X \mid X<\lambda^{*}\right]$ remains constant. Clearly, Conditions (i) and (ii) together imply that $E\left[X \mid X<\lambda^{*}\right]$ is constant, but the converse is not true.

3 In this case, the second-order condition, $H^{\prime \prime}\left(\lambda^{*} ; u, F, z\right)<0$, does not necessarily hold for all $\lambda^{*} \in(0, L)$. However, sufficient conditions for $H^{\prime \prime}\left(\lambda^{*} ; u, F, z\right)<0$ can be found by making the insured's risk aversion coefficient, $r(\bullet)=-u^{\prime \prime}(\bullet) / u^{\prime}(\bullet)$, sufficiently large. Details are provided in a technical appendix available from the authors. 
In short, Theorem 2 states that if the risk is transformed in a manner that preserves the mean loss below the policy limit and is stochastically equivalent at the policy limit, then at least as much insurance will be purchased if and only if the transformation yields a better risk, in the first-order sense, above the policy limit.

As with deductibles, transformations of the loss distribution are often exogenous to the policy limit selected by the insured, and so it is not clear that the transformations would necessarily be mean-preserving below the insured's particular policy limit. However, this condition certainly would hold for problems involving statutory or regulatory changes that affect the insured's obligation to pay relatively large losses (i.e., those clearly above the limit). For example, any change in federal or state tort liability law that has the effect of restricting the insured's responsibility for paying the portion of any liability loss $X$ that is greater than $\lambda^{\prime}$ dollars (where $\lambda^{\prime}>\lambda^{*}$ ) would satisfy the conditions of Theorem 2 .

Finally, as with deductibles, it is quite likely that any risk transformation arising from risk management activities that are endogenous to the insured would depend specifically on the insured's policy limit.

\section{Directions for Further Research}

In this study, the authors have identified both necessary and sufficient conditions for unambiguous comparative statics for insurance demand for both coverage deductibles and policy limits.

One important finding of this research is that, for the case of insurance deductibles, it is natural to consider transformations of the loss distribution that are mean-preserving above the optimal deductible, whereas for policy limits, it is natural to consider transformations of the loss distribution that are mean-preserving below the optimal limit. This is because an MPT for the entire distribution may affect insurance-purchasing behavior more by a change in price than by any change in risk.

As noted above, in cases where the risk transformation arises from risk management activities that are clearly endogenous to the insured, it is quite likely that the transformation would depend specifically on the insured's particular deductible and/or policy limit. However, models of this type generally would require consideration of an additional decision variable reflecting the amount of resources directed to the risk management program. In ongoing research, the authors plan to address the issue of multiple decision variables, with special attention to the tradeoffs between market insurance and self-insurance.

Beyond additional modeling, another next step would be to test the preceding comparative static results with empirical data from one or more insurance markets, with property insurance the most likely candidate. However, in carrying out any empirical analysis of real-world markets, two important supply-side issues must be addressed: (1) whether the assumption of actuarially based pricing under all transformations of the loss variable is reasonable and (2) to what extent the policy parameters $\delta$ and $\lambda$ are dictated by the risk preferences of insurers, rather than insureds, in market equilibrium. 


\section{Appendix}

\section{Proof of Theorem 1:}

Given that the transformation $F(\bullet) \rightarrow G(\bullet)$ is both mean-preserving above $\delta^{*}$ and stochastically equivalent at $\delta^{*}$, it can be seen that $p=(1+\theta) E\left[\operatorname{Max}\left\{X-\delta^{*}, 0\right\}\right]$ and $\frac{d p}{d \delta^{*}}=-(1+\theta) \operatorname{Pr}\left\{X>\delta^{*}\right\}$ are unaffected by the change in the loss distribution.

Now consider the difference

$$
\begin{aligned}
H^{\prime} & \left(\delta^{*} ; u, G, z\right)-H^{\prime}\left(\delta^{*} ; u, F, z\right) \\
= & (1+\theta)\left[1-F\left(\delta^{*}\right)\right] \int_{0}^{\delta^{*}} u^{\prime}(W-x-p)[d G(x)-d F(x)] \\
& +\left\{(1+\theta)\left[1-F\left(\delta^{*}\right)\right]-1\right\} u^{\prime}\left(W-\delta^{*}-p\right) \int_{0}^{\delta^{*}}[d G(x)-d F(x)] \\
= & (1+\theta)\left[1-F\left(\delta^{*}\right)\right] F\left(\delta^{*}\right) \int_{0}^{\delta^{*}} \frac{u^{\prime}(W-x-p)}{F\left(\delta^{*}\right)}[d G(x)-d F(x)]+0 \\
= & (1+\theta)\left[1-F\left(\delta^{*}\right)\right] F\left(\delta^{*}\right) \\
& \times\left\{E_{G}\left[u^{\prime}(W-X-p) \mid X<\delta^{*}\right]-E_{F}\left[u^{\prime}(W-X-p) \mid X<\delta^{*}\right]\right\} .
\end{aligned}
$$

From standard properties of first-order stochastic dominance, it follows that, for a function $u^{\prime}(W-X-p)$ that is increasing in $X$, the difference

$$
E_{G}\left[u^{\prime}(W-X-p) \mid X<\delta^{*}\right]-E_{F}\left[u^{\prime}(W-X-p) \mid X<\delta^{*}\right]
$$

and therefore the entire right side of Equation (A1), is nonpositive if and only if Condition (3) holds. Given the first-order condition [Equation (2)], we then see that

$$
H^{\prime}\left(\delta^{*} ; u, G, z\right)-H^{\prime}\left(\delta^{*} ; u, F, z\right)=H^{\prime}\left(\delta^{*} ; u, G, z\right) \leq 0,
$$

and so the insured will purchase at least as much insurance under $G(\bullet)$ as under $F(\bullet)$.

\section{Proof of Theorem 2:}

The proof of this theorem is analogous to that of Theorem 1.

Given that the transformation $F(\bullet) \rightarrow G(\bullet)$ is both mean-preserving below $\lambda *$ and stochastically equivalent at $\lambda *$, it can be seen that $p=(1+\theta) E[\operatorname{Min}\{X, \lambda *\}]$ and

$$
\frac{d p}{d \lambda^{*}}=(1+\theta) \operatorname{Pr}\left\{X>\lambda^{*}\right\}
$$

are unaffected by the change in the loss distribution. 
Now consider the difference

$$
\begin{aligned}
H^{\prime}\left(\lambda^{*} ; u, G, z\right)-H^{\prime}\left(\lambda^{*} ; u, F, z\right) \\
=-(1+\theta)\left[1-F\left(\lambda^{*}\right)\right] u^{\prime}(W-p) \int_{0}^{\lambda^{*}}[d G(x)-d F(x)] \\
+\left\{1-(1+\theta)\left[1-F\left(\lambda^{*}\right)\right]\right\} \int_{\lambda^{*}}^{L} u^{\prime}\left(W-x+\lambda^{*}-p\right)[d G(x)-d F(x)] \\
=0+\left\{1-(1+\theta)\left[1-F\left(\lambda^{*}\right)\right]\right\}\left[1-F\left(\lambda^{*}\right)\right] \int_{\lambda^{*}}^{L} \frac{u^{\prime}\left(W-x+\lambda^{*}-p\right)}{\left[1-F\left(\lambda^{*}\right)\right]}[d G(x)-d F(x)] \\
=\left\{1-(1+\theta)\left[1-F\left(\lambda^{*}\right)\right]\right\}\left[1-F\left(\lambda^{*}\right)\right] \\
\quad \times\left\{E_{G}\left[u^{\prime}\left(W-X+\lambda^{*}-p\right) \mid X>\lambda^{*}\right]-E_{F}\left[u^{\prime}\left(W-X+\lambda^{*}-p\right) \mid X>\lambda^{*}\right]\right\} .
\end{aligned}
$$

Since $\theta<\frac{F\left(\lambda^{*}\right)}{1-F\left(\lambda^{*}\right)}$ [from the first-order condition, Equation (5)], it follows from standard properties of first-order stochastic dominance that, for a function $u^{\prime}\left(W-X+\lambda^{*}-p\right)$ that is increasing in $X$, the difference

$$
E_{G}\left[u^{\prime}\left(W-X+\lambda^{*}-p\right) \mid X>\lambda^{*}\right]-E_{F}\left[u^{\prime}\left(W-X+\lambda^{*}-p\right) \mid X>\lambda^{*}\right],
$$

and therefore the entire right side of Equation (A2), is nonnegative if and only if Condition (6) holds. Given Equation (5), we then see that

$$
H^{\prime}\left(\lambda^{*} ; u, G, z\right)-H^{\prime}\left(\lambda^{*} ; u, F, z\right)=H^{\prime}\left(\lambda^{*} ; u, G, z\right) \geq 0
$$

and so the insured will purchase at least as much insurance under $G(\bullet)$ as under $F(\bullet)$

\section{References}

Black, J. M., and G. Bulkley, 1989, A Ratio Criterion for Signing the Effects of an Increase in Uncertainty, International Economic Review, 30(1): 119-130.

Briys, E., G. Dionne, and L. Eeckhoudt, 1989, More on Insurance as a Giffen Good, Journal of Risk and Uncertainty, 2(4): 415-420.

Diamond, P. A., and J. E. Stiglitz, 1974, Increase in Risk and in Risk Aversion, Journal of Economic Theory, 8(3): 337-360.

Dionne, G., and L. Eeckhoudt, 1987, Proportional Risk Aversion, Taxation, and Labor Supply Under Uncertainty, Journal of Economics, 47(4): 353-366.

Dionne, G., and C. Gollier, 1992, Comparative Statics Under Multiple Sources of Risk With Applications to Insurance Demand, Geneva Papers on Risk and Insurance Theory, 17(1): 21-33.

Dreze, J., and F. Modigliani, 1972, Consumption Decisions Under Uncertainty, Journal of Economic Theory, 5(3): 308-335. 
Eeckhoudt, L., and P. Hansen, 1980, Minimum and Maximum Prices, Uncertainty and the Theory of the Competitive Firm, American Economic Review, 70(5): 1064-1068.

Eeckhoudt, L., and P. Hansen, 1983, Micro-Economic Applications of Marginal Changes in Risk, European Economic Review, 22(2): 167-176.

Eeckhoudt, L., C. Gollier, and H. Schlesinger, 1991, Increases in Risk and Deductible Insurance, Journal of Economic Theory, 55(2): 435-440.

Gollier, C., 1995, The Comparative Statics and Changes in Risk Revisited, Journal of Economic Theory, 66(2): 522-535.

Meyer, J., and M. B. Ormiston, 1983, The Comparative Statics of Cumulative Distribution Function Changes for the Class of Risk Averse Agents, Journal of Economic Theory, 31(1): 153-169.

Meyer, J., and M. B. Ormiston, 1985, Strong Increases in Risk and Their Comparative Statics, International Economics Review, 26(2): 425-437.

Meyer, J., and M. B. Ormiston, 1999, Analyzing the Demand for Deductible Insurance, Journal of Risk and Uncertainty, 18(3): 223-230.

Rothschild, M., and J. Stiglitz, 1970, Increasing Risk I: A Definition, Journal of Economic Theory, 2(3): 225-243.

Rothschild, M., and J. Stiglitz, 1971, Increasing Risk II: Its Economic Consequences, Journal of Economic Theory, 3(1): 66-84.

Schlesinger, H., 1981, The Optimal Level of Deductibility in Insurance Contracts, Journal of Risk and Insurance, 48(3): 465-481. 